\title{
Nuclear emulsion techniques for muography
}

\author{
Cristiano Bozza ${ }^{1,2,{ }^{\star}}$, Lucia Consiglio ${ }^{3,4}$, Nicola D’Ambrosio ${ }^{5}$, Giovanni De Lellis ${ }^{3,4}$, \\ Chiara De Sio ${ }^{1,2}$, Seigo Miyamoto ${ }^{6}$, Ryuichi Nishiyama ${ }^{6}$, Chiara Sirignano ${ }^{7,8}$, \\ Simona Maria Stellacci ${ }^{1,2}$, Paolo Strolin ${ }^{3,4}$, Hiroyuki K.M. Tanaka ${ }^{6}$, Valeri Tioukov ${ }^{3,4}$
}

\author{
${ }^{1}$ Università di Salerno, Dipartimento di Fisica “E. R. Caianiello", Salerno, Italy \\ ${ }^{2}$ Istituto Nazionale di Fisica Nucleare (INFN), Gruppo Collegato di Salerno, Salerno, Italy \\ ${ }^{3}$ Università di Napoli Federico II, Dipartimento di Fisica, Naples, Italy \\ ${ }^{4}$ Istituto Nazionale di Fisica Nucleare (INFN), Sezione di Napoli, Naples, Italy \\ ${ }^{5}$ Istituto Nazionale di Fisica Nucleare (INFN), Laboratori Nazionali del Gran Sasso, Assergi (L'Aquila), Italy \\ ${ }^{6}$ Earthquake Research Institute, The University of Tokyo, Tokyo, Japan \\ ${ }^{7}$ Università di Padova, Dipartimento di Fisica e Astronomia “Galileo Galilei”, Padova, Italy \\ ${ }^{8}$ Istituto Nazionale di Fisica Nucleare (INFN), Sezione di Padova, Padova, Italy
}

\author{
Article history \\ Received September 15, 2016; accepted October 5, 2016. \\ Subject classification: \\ Muography, Muon radiography, Nuclear emulsion, Volcano radiography, Cosmic rays.
}

\begin{abstract}
Nuclear emulsions are currently being used in the field of muography, more specifically muon radiography of volcanic edifices and fault regions. The peculiar features of such detector for cosmic muons demand appropriate data processing and analysis techniques. The paper shows the current development status of readout devices and analysis techniques developed by some research groups that established a collaborative network in Italy and Japan. An overview is given of nuclear emulsion-based detectors, from the detection principles to detector operation and set-up techniques, in connection with the expectations in terms of geophysics information. Two systems for readout are presented, one developed in the first decade of the 21st century and one that is entering duty now. The evolution in terms of data quality and speed is discussed. Finally, the most relevant data processing steps that allow working out muon absorption maps from nuclear emulsion data are described.
\end{abstract}

\section{Introduction}

Nuclear emulsions have gained increasing interest over the last years as particle detectors for muon radiography (see e.g. Tanaka et al. [2007a]). Emulsions have little or no alternative when a power supply is not available in the detector setup station, which is relatively likely to occur; furthermore, the cost of emulsion films to span a large area $\left(1 \mathrm{~m}^{2}\right.$ or more $)$ is much lower than the cost of complex electronic detectors. As shown in the following sections, there are now readout systems that have been designed explicitly for this research field. The driving concepts for detector design and operation in the field of muon radiography can be summarized as follows:

- the measured muon flux is always binned in a $2 \mathrm{D}$ angular histogram;

- consequently, the minimum size of the structures that can be imaged (magma chambers, conduit, faults, cracks) is inversely proportional to the square root of the available statistics in the angular region of interest, because a difference in absorption between two neighbouring angular bins should be statistically significant;

- the energy spectrum of muons from cosmic rays is relatively soft, and a very small fraction of muons has enough energy to travel across large structures $(500 \mathrm{~m}$ or more);

- consequently, the thicker the edifice or fault region, the longer the exposure time needed to image fine details;

- as a second consequence of the muon spectrum, real-time or quasi-real-time monitoring of volcano eruptions by observation of the motion of magma is possible only in shallow craters or conduits but not indepth, regardless of the detector used;

- instrumental or physical background must be kept under control in particular in the region of low statistics (typically low-elevation, observing horizontal muons through the bulk of volcano edifices).

The flow of raw data is found to be huge and comparable to medium-large high-energy physics experi- 


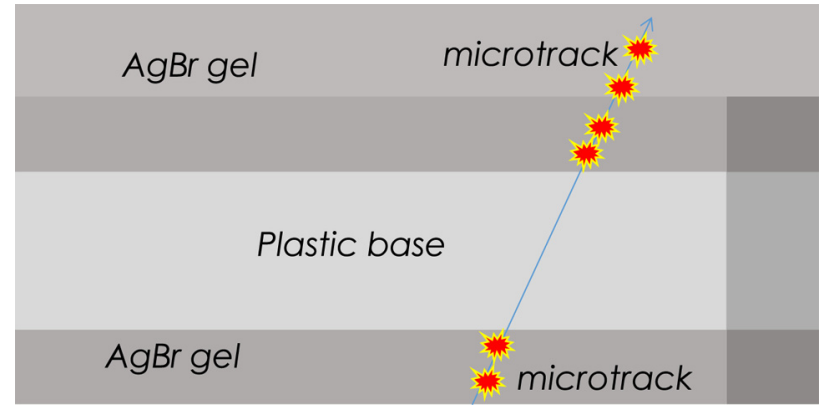

Figure 1. Structure of a typical emulsion film with double coating on a plastic base. Microtracks are formed in the sensitive gel by ionising tracks. Pairs of microtracks can be connected across the base to build base-tracks.

ments. Data reduction is applied in several steps and the final result is the muon absorption map. Full software chains are available to work out the predicted shape of the absorption profile and compare it to the output of data processing. Developments in the technique are in progress also for what concerns simulation and data analysis.

\section{Nuclear emulsion detectors}

The technology and principles of nuclear emulsions are similar to photographic emulsions. While the latter are used to detect photons and form an image, the former are used to detect ionising particles passing through a medium by recording a 3D track. Nuclear emulsions are usually made of $\mathrm{AgBr}$ crystals interspersed in a gel (often of animal origin). An ionising particle would sensitise the crystals traversed leaving what is called a latent image (Figure 1). The process of development (similar to that of photographic emulsions) makes metallic Ag coalesce onto the sensitised site, growing folded filaments of the order $100 \mathrm{~nm} \sim 1 \mu \mathrm{m}$. After development is complete and the image is fixed, clusters of such filaments, called grains, are observed in transmitted light undergoing diffractive scattering and appear as dark spheroids on a light background. Grains are aligned along the path of the particle in the medium, which makes nuclear emulsions a natively $3 \mathrm{D}$ tracking detector, usually with a sub-micrometric precision, two qualities that no other detection technology can offer. Even a very thin layer of emulsion $(20 \sim 30 \mu \mathrm{m})$ can give 3D tracks with angular precision of the order of $10 \mathrm{mrad}$. On the other hand, nuclear emulsions are sensitive since their production to the time they are developed. Thermal effects and humidity slowly delete the latent image, which is even more sensitive to chemical reactants. As a result, a developed emulsion shows the record of all the ionising particles that crossed it, including those due to natural radioactivity in storage sites, and ambient and cosmic radiation during transport to the exposure site. In other words, there is no time trigger (unlike elec- tronic detectors) and tracks born in different events may be geometrically very close-by without having any physical relationship. Nuclear emulsion films are commonly used in double-coating configuration, with a transparent plastic base coated on both sides with sensitive emulsion layers. In this case, each ionising particle crosses both sides, providing two sequences of aligned grains, called microtracks.

As applied to the field of muon radiography, the peculiarities of emulsion have a direct impact on the data analysis techniques and possible results. The net counting of tracks through a film in an angular region is related in a non-trivial way with the expected muon flux: in an electronic detector the shower accompanying a muon produces a single trigger with itself, whereas in an emulsion-based detector the absence of time resolution and triggering functions flattens out the exposure timeline. The precision of spatial measurements can be exploited to identify particles and to estimate their momenta, washing away the soft components in the muon shower and ideally leaving only the muon (Figure 2). The average deviation of grains of a hard muon ( $1 \mathrm{GeV}$ and higher) from the straight line of the fit, in absence of magnetic field and with negligible scattering, is of the order of $50 \mathrm{~nm}$. Even including distortion effects, scattering and stray magnetic fields, a track can usually be measured with sub-micrometric precision. Nuclear emulsion has usually a density about $3 \mathrm{~g} / \mathrm{cm}^{3}$, with a non-negligible stopping power, especially for soft components and electrons. The energy loss of charged particles for ionisation and bremsstrahlung can be increased by interleaving emulsion films with plates of high $\mathrm{Z}$ material, such as iron or lead. Multi-

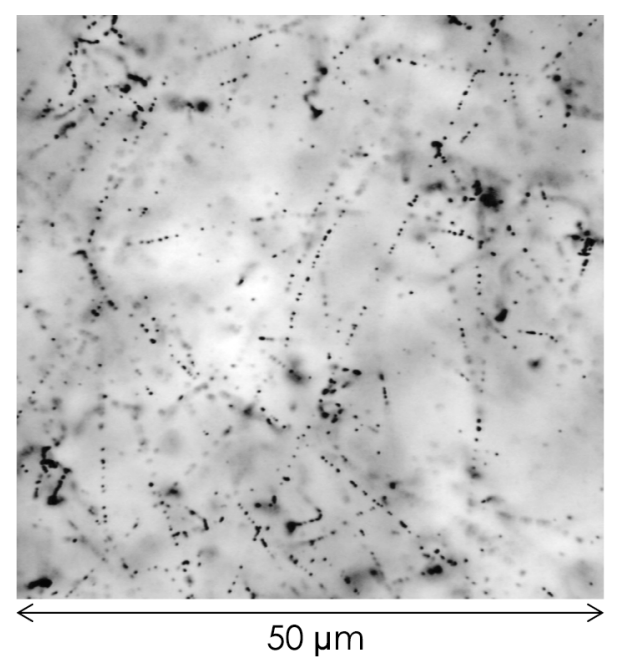

Figure 2. Emulsion image in transmitted light. Straight tracks can be seen, due to vertically incoming cosmic rays. Typically, emulsion films are exposed orthogonally to the direction of muons traversing the volcanic edifice, so a single dark grain is seen for a track in each focussed plane. 


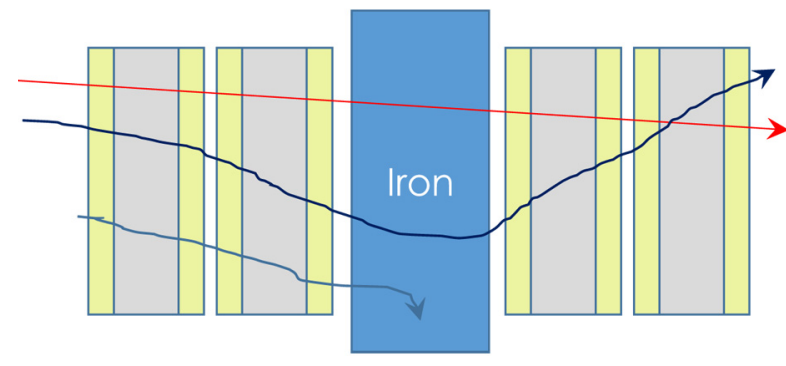

Figure 3. Detector made of stacked nuclear emulsion films with an iron absorber. Each film is sketched as a pair of sensitive $\mathrm{AgBr}$ layers on a plastic base. Hard muons go straight through the stack. Soft muons are scattered in the absorber or may be stopped.

ple Coulomb scattering becomes much stronger than just a disturbance in grain alignment, giving rise to sizable deflections (from several mrad to $100 \mathrm{mrad}$ or more; or, from few $\mu \mathrm{m}$ to $100 \mu \mathrm{m}$ or more), inversely correlated with the momentum of the deflected charged particle (Figure 3) [Agafonova et al. 2012]. In addition, electrons and positrons travelling in a dense medium radiate photons which in turn convert to pairs. Electromagnetic showers show up in emulsion accompanying hard muons.

Detectors based on nuclear emulsions have a modular structure. The largest films ever produced for largescale application did not reach $1 \mathrm{~m}$ per dimension and usually the typical size is about $10 \mathrm{~cm}$ (see e.g. Nakamura et al. [2006]). Collecting a high statistics of cosmic ray muons in reasonable time (i.e. few months' campaigns) requires at least $1 \mathrm{~m}^{2}$ (but studies are in progress to build $10 \mathrm{~m}^{2}$ and $100 \mathrm{~m}^{2}$ detectors); hence several unit films are needed to cover the whole cross section of the detector. In addition, films are stacked to improve angular precision and allow particle identification and kinematical measurements. In practice, a cosmic ray muon goes through $8 \sim 100$ emulsion films while crossing the detector from side to side. In order to filter out the electromagnetic (electrons/positrons) and soft component of the spectrum (muons with $p<1 \mathrm{GeV} / \mathrm{c}$ ) one can add metal absorbers before and after the detector and by stacking films interleaved with spacers or dense scattering material. In the latter case, as pointed out above, the multiple Coulomb scattering effect can be computed precisely, and soft tracks can be rejected on the basis of their estimated momentum, by properly adjusting the tolerances for track connection.

Finally, it must be kept in mind that nuclear emulsions are hygroscopic and sensitive to temperature and humidity conditions. The usual working range is $4 \sim 30^{\circ} \mathrm{C}$. Higher temperatures cause melting of the emulsion, whereas low temperature affect their sensitivity. This limits data taking campaigns to cool periods (excluding hot summers where they occur). Care is needed during emulsion transportation to the exposure place and during detector removal: since nuclear emulsions are always sensitive until developed, they pile up tracks also during transportation. Two policies are possible: either the stacking order is completely reshuffled soon before exposure and at the end of the exposure, so that track alignment is destroyed; or the stacking order is reversed, so that it is possible by off-line data processing to recognize which tracks are due to transportation times and which were produced during exposure (Virtual Erasing). A careful treatment of background (see e.g. Nishiyama et al. [2014]) is typically needed when muographic exposures have to probe large objects ( $>500 \mathrm{~m}$ in rock) and/or at low elevation. Indeed, with very few hard muons to be detected, instrumental and physical backgrounds add fake counts (even of the order of 10 tracks $/ \mathrm{cm}^{2}$ ), biasing the statistics with an excess of muons, which in turn leads to underestimating the average rock density.

Nuclear emulsions are cheap (a few $€ /$ film) and require no power. Therefore, they turn out to be ideal for long-term campaigns in places where electric power supply is not available.

\section{Readout devices}

The major progress of nuclear emulsion technology in the last decades is the massive automation of readout. For the pioneer work, see Niwa et al. [1974] and Aoki et al. [1990]. In $100 \mathrm{~cm}^{2}$ there may be $10^{6}$ tracks whose positions and slopes have to be recorded. This is clearly out of reach for readout by human operators, but is a typical application field for computerdriven machines. The optical microscopes that were used in the past have been replaced with Cartesian robots with a readout head (high-resolution, high-speed camera) mounted on the optical axis. Within each field of view, the optical axis can translate onto itself to slice the volume into tomographic sequences of $2 \mathrm{D}$ images, in which the dark (metallic Ag) grains appear on a light background. The $\mathrm{X}$ and $\mathrm{Y}$ coordinates are given by the positions of dark pixels in the image, whereas the $Z$ coordinate is inferred from the slice number. The position information collected for each grain is hence three-dimensional, which allows track reconstruction by recognizing 3D alignments of grains. Readout heads can take data from much less than $1 \mathrm{~mm}^{2}$ in one view. For this reason, the XY stage needs to be able to move to scan the whole surface of the film. This is a good reason to avoid very large films: the inertia of the XY stage should not be so large as to prevent good mechanical performances, and this puts a practical limitation on the size of films. In the following, two readout systems for muography research are presented as concrete examples. Other 
existing systems may have some parts in common with them or not, but the basic principles are the same.

The European Scanning System (ESS) [Armenise et al. 2005, De Serio et al. 2005, Arrabito et al. 2006, Arrabito et al. 2007, Bozza et al. 2013] was built in the framework of high-energy physics for the OPERA experiment [Acquafredda et al. 2009, Agafonova et al. 2014] inheriting basic ideas from the SySal system [Rosa et al. 1997], used in the late 90's in the CHORUS experiment [Eskut et al. 1997, 2008] at CERN. In its most common configuration, the ESS was built with a MICOS XY stage ( $0.1 \mu \mathrm{m}$ precision) equipped with a plate holder, a linear MICOS stage for the $Z$ axis $(0.05$ $\mu \mathrm{m}$ precision) holding the optical tube on which the objective and the camera were hosted. Illumination was produced in transmitted mode with the beam parallel to the $Z$ axis by another centred optical system. The most commonly used objective has $50 \times$ magnification. In these conditions, the camera (Mikrotron MC1310), with 1.2 MPixel resolution (grey scale), has a field of view with linear dimensions of about $400 \times 320 \mu \mathrm{m}^{2}$, but about $30 \mu \mathrm{m}$ overlap between adjacent fields of view is used to make sure that tracks appear as a whole at least in one view. 15 images per field of view are grabbed at 376 frames per second and transferred via Camera Link to a vision processor (Matrox Odyssey) that performs image processing (lighting equalization, dark pixel detection, binarization) sending data to the host CPU to perform the final step of 2D image recognition. 3D alignment of grains to recognize tracks can be performed on the workstation or by a cluster of "tracking servers". The host workstation is equipped with a motion controller (National Instruments FlexPCI 7344) to drive the stage axes while images are grabbed on the fly. This system implements a "stop and go" motion on the $\mathrm{X}$ axis, i.e. while the $\mathrm{Z}$ axis descends to get the tomographic images the $\mathrm{X}$ axis is kept hold- ing steady, and moves to the next field of view when the $Z$ axis resets to the start position for a new tomography. Despite the considerable accelerations that are needed to run the data-taking cycle in $185 \mathrm{~ms}$, mechanics is not the bottleneck of this system: the overall pace is actually set by the processing speed of the Matrox Odyssey. The resulting data taking speed is 20 $\mathrm{cm}^{2} / \mathrm{h} /$ side of film, but in muography applications data taking speed is closer to $16 \mathrm{~cm}^{2} / \mathrm{h} /$ side because 21 images are acquired to allow for non-planarity of the film over large areas.

The Quick Scanning System (QSS; see Figure 4) is a major upgrade of the ESS, sharing with it only the mechanical base (i.e. the MICOS stages) and the illumination system (which could in principle be replaced with any other similar component). The QSS was designed to be used in the field of muography, making a speed leap while keeping cost under control. For example, at a readout speed of $150 \mathrm{~cm}^{2} / \mathrm{h}$ (about 10 times faster than the average speed of ESS), and assuming 2 systems with $100 \%$ operation rate, the overall data taking speed can reach $150 \mathrm{~cm}^{2} / \mathrm{h}$, which translates to 10.8 $\mathrm{m}^{2} /$ month. After exposure and development the density image of Showa-Shinzan lava dome [Tanaka et al. 2007b] would be obtained in less than 2 days.

This system has even greater flexibility with respect to components, and its evolution is continuing at the time of the present paper. Different installations have recorded scanning speed as high as $43 \mathrm{~cm}^{2} / \mathrm{h} /$ side, $65 \mathrm{~cm}^{2} / \mathrm{h} /$ side and $91 \mathrm{~cm}^{2} / \mathrm{h} /$ side with 31 images/view sampling, depending mostly on the version of software used to drive data taking and mechanics. The motion controller is currently Pro-Dex OMS MAXk4000/5000. Several cameras have been tested, and at the moment the most common solution is to use CMC 4000, capable of about 4 MPixel at $385 \mathrm{fps}$, which means roughly increasing the peak data rate by a factor 4 with respect

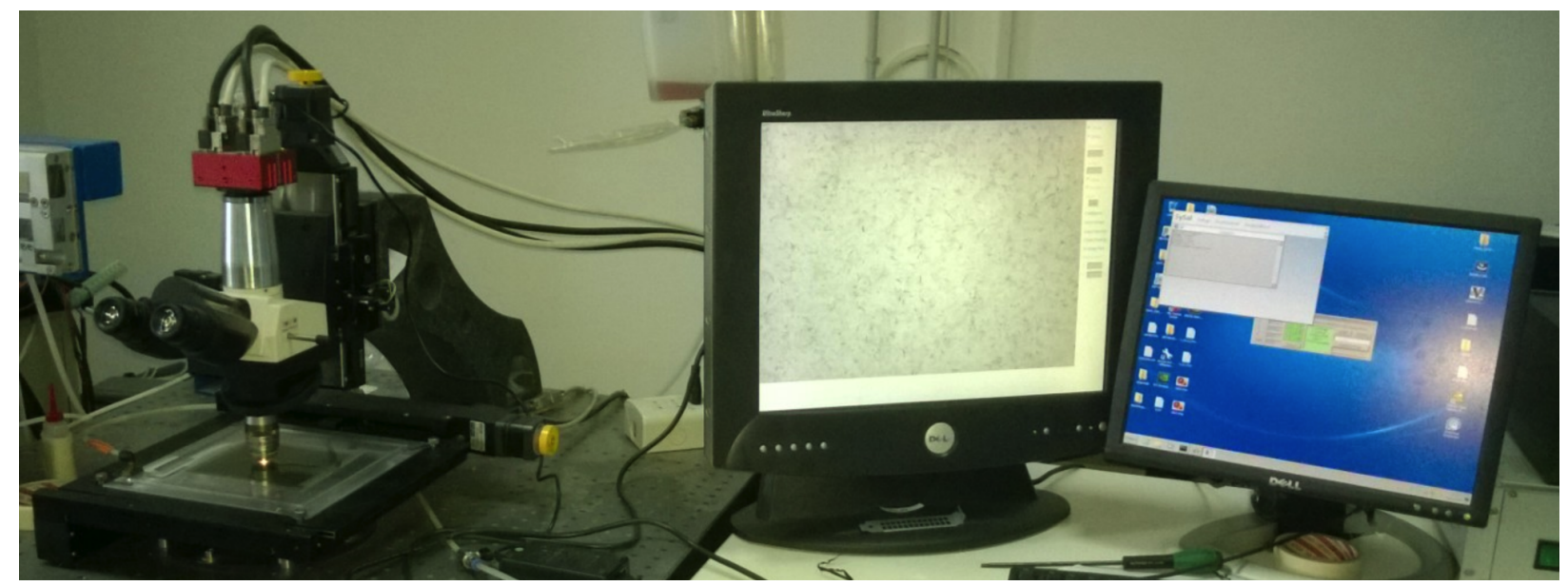

Figure 4. The QSS setup. A Cartesian robot with readout head (automatic microscope) is seen on the left. In the centre and right part of the picture, screenshots of the data acquisition program, showing a live image of the emulsion. 
to ESS. The optics is adjusted to get a resolution that is similar to the one of ESS. Images are grabbed by Matrox Radient, a new generation frame grabber, which is however used only to transfer images to the host memory. Image processing is performed by using GPU's. The NVidia GTX series boards 590, 690 and Titan Black have all been successfully tested to work with the QSS. Tracking is off-loaded to a factory of GPU-based tracking servers, which can run virtually any NVidia board (GTX 640, Tesla C2050, GTX 590, GTX 690, GTX 780 Ti and GTX Titan Black have been tested). Another major improvement in speed comes from the stage drive technique, which is called continuous motion: the $\mathrm{X}$ (or $\mathrm{Y}$ ) axis is set to move at constant speed, while the $\mathrm{Z}$ axis cyclically descends during image acquisition and resets to the starting position. This implies that images are taken with the stage in motion: a short image gate (usually $24 \mu \mathrm{s}$ ) and strong illumination are used to get nearly still images, which then need considerable computing power to be realigned and merged to build long $\mathrm{X}(\mathrm{Y})$ strips from the skewed volumes that correspond to each field of view. With the present hardware, the QSS aims at $120 \mathrm{~cm}^{2} / \mathrm{h} /$ side. Speed between 150 and $180 \mathrm{~cm}^{2} / \mathrm{h} /$ side could be achieved by sampling $21 \mathrm{im}$ ages instead of 31. It is worth to mention that the cost of the upgrade from ESS to QSS is about $1 / 4$ of the cost of the ESS.

\section{Data processing: from raw data to muon absorption maps}

The treatment of data from automatic readout systems for the purposes of muography can be summarized in a single sentence as a progressive reduction from local measurements to a muon absorption map in the slope plane (detector plane reference) or angular plane (direction and elevation). This very intuitive overview is a useful guideline to keep in mind while all the steps are described.

The basic pieces of a muon track are the abovementioned microtracks, i.e. sequences of grains aligned along a straight line in a single emulsion layer. In most cases, emulsion films have a double coating around a plastic base, so it is relatively easy to recognize pairs of microtracks corresponding to the same track. This step is sometimes called base-track formation as the pairs of microtracks connected across the base are called basetracks. It is not uncommon to have $10^{3} \sim 10^{4}$ microtracks $/ \mathrm{mm}^{2}$, most of which are due to ambient radioactivity and therefore correspond to soft electrons not crossing the base. Base-track formation improves the signal/background ratio for high-energy muons by at least two orders of magnitude, decreasing also the amount of data to be handled by the next steps. On the

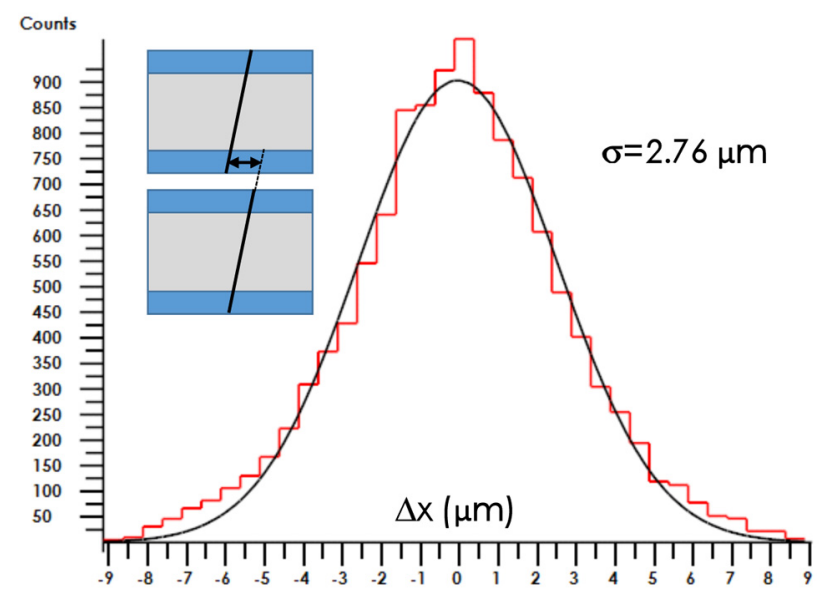

Figure 5. Film-to-film alignment of base-tracks to form volumetracks. As displayed in the sketch, each base-track on one film is projected using its position and slope to the other film. Films are in contact without spacers. The difference between the projected position and the position of the connected base-track is shown.

other hand, it decreases the overall efficiency, because a microtrack will be discarded if its partner on the other side of the base is not found. Denoting the efficiency for micro-track recognition as $\epsilon_{m}$ and the efficiency after base-track formation with $\epsilon_{b}$, one can write:

$$
\epsilon_{b} \leq \epsilon_{m}
$$

A hard muon will cross several films if they are stacked, producing a set of aligned base-tracks. A volume-track is made of several base-tracks connected in consecutive films. Depending on the setup, volumetracks might have different requirements. With many films in the stack, it becomes convenient to allow also for missing base-tracks, otherwise the overall efficiency would become as low as $\epsilon_{m}^{f}$, fbeing the number of twosided films. For example, in a quadruplet setup, typically triplet and quadruplet volume-tracks are used to estimate the real number of crossing tracks.

Secondary cosmic radiation has a high content of electrons/positrons and soft muons. If plates or slabs of high-Z, high-density absorbers, such as iron or lead, are interleaved with the emulsion films, it is possible to cause electrons / positrons to start electromagnetic showers and "disappear" in consecutive films; by applying proper alignment tolerances, it is possible to reject soft muons. Figure 5 shows the results for hard muons. As anticipated above, small fluctuations in the thickness of material traversed by such soft components, which constitutes the large majority of all tracks, can bring problems in the absolute flux normalization; furthermore, statistical fluctuations of such dominant component would mask local density anomalies, whose detection is the goal of muography. The remaining population of tracks after the cuts consists mostly of hard muons, 


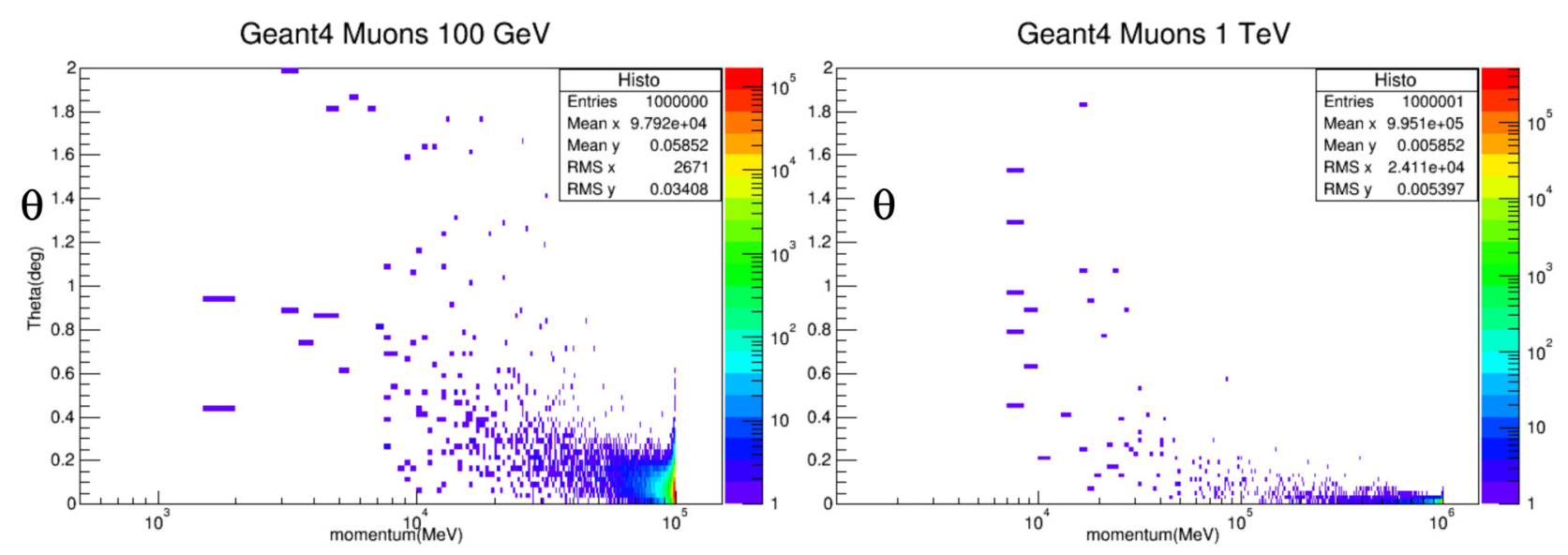

Figure 6. Simulation of the propagation of muons through $2 \mathrm{~m}$ rock slabs in Geant4. The distributions of the deflection angle and residual momentum at the exit point of the slab are shown for $100 \mathrm{GeV}$ and $1 \mathrm{TeV}$ muons.

whose statistics is related to the total thickness and the averaged density of rock traversed, with typical dynamic ranges of $10 \sim 1000 \mathrm{~m}$ in standard rock.

Volume-tracks have even better angular precision than base-tracks. It is common, at the end of processing, to have tracks with aiming accuracy of the order of $1 \mathrm{mrad}$. If the distance between the detection station and an anomaly being measured is of the order of 500 $\mathrm{m}$, this means that the intrinsic resolution provided by the emulsion detector is about $0.5 \mathrm{~m}$. Such resolution is well suited to observe conduits and cracks, but it is to be understood as a lower limit. The real resolution depends on the available statistics and on the relative density variation of anomalies. In realistic setups, the muon absorption map is built with at least $10^{-3} \mathrm{sr}$ bins, or about $10 \mathrm{mrad}$ per projection (X-Y in the detector reference), or $0.5^{\circ}$ direction/ elevation. In regions with low elevation, and hence high thickness $(500 \mathrm{~m}$ or more) and low passing-through flux, bins may be even 10 times bigger to detect fluctuations with at least $2 \sigma$ significance. As a consequence, the effective resolution is better in shallow regions (e.g. volcano crater) and worsens as the elevation decreases.

Detecting small anomalies requires high statistics, which in turn means high processing power. Image data in a single film $\left(120 \mathrm{~cm}^{2}\right)$ commonly top $3 \mathrm{~TB}$; microtrack data fit normally in $30 \mathrm{~GB} /$ film; correspondingly, base-tracks take about $1.5 \mathrm{~GB} /$ film, and volume-tracks take $400 \mathrm{MB}$ in a quadruplet geometry if only triplet and quadruplet tracks are kept. For $1 \mathrm{~m}^{2}$ detector, the amount of data at the end of processing is about $40 \mathrm{~GB}$. For data-taking campaigns that have been recently completed, upgrades of one or two orders of magnitudes are envisaged.

Simulation of the expected flux is also being considered as an area for improvement and evolution. Both the secondary cosmic muon flux and the passage of such particles through matter are receiving attention.
At the present time, the muon flux impinging on the volcanic edifice is obtained by interpolating tabulated results found in the literature, but these are known to suffer from large systematic errors in the high-energy tail of the muon spectrum, which contains the only muons that are able to traverse large thickness of rock (500 $\mathrm{m}$ and more). The usage of software tools (in particular COSMOS and CORSIKA) that perform calculations starting from the flux of primary cosmic particles and simulate their propagation and interactions through the atmosphere is being explored.

The propagation of muons in matter is currently being simulated by the continuous slowing down approximation (CSDA), i.e. the fluctuations due to the stochastic nature of energy loss in matter are not being considered. Indeed these are relevant in the region of low elevation and large thickness, because a relatively long tail in the range distribution might lead to an overestimation of the flux and a consequent underestimation of the average density. Furthermore, the effect of direction change on scattered muons is expected to give a sizable contributions for directions close to the mountain profile, where muons might be bent "inwards" and migrate to inner angular bins. This effect should be negligible for large binning or energies above $10 \mathrm{GeV}$, but should be taken into account elsewhere. Muon propagation tables are being obtained by full Geant 4 simulations of muons traversing rock slabs; they should be plugged into the cosmic muon propagation software that receives the mountain geometry and works out the expected binned flux.

\section{Conclusions}

The paper presented a short but up-to-date report of the technique of nuclear emulsions applied to muon radiography of volcanic edifices. Emulsion-based detectors are cheap, simple and versatile to operate. The lack of time trigger is more than compensated in many 
cases by the unequalled spatial resolution. The basic concepts of readout systems have been shown, and two systems, the ESS and the QSS, have been discussed in detail. The progress in scanning speed will allow increasing the statistics of tracks in muographic data-taking campaigns. The consequent improvements in signal/noise ratio and in the statistical significance of possibly detectable anomalies are evident. Data processing techniques profit of the unique features of nuclear emulsions. Their evolution is twofold: on one hand, the increase in data rate drives the need for highspeed data processing; on the other hand, more sophisticated simulations are starting to consider the models of secondary cosmic rays and the details of their propagation through matter.

Muography by nuclear emulsions is now an established investigation field. While the technique of nuclear emulsions themselves is very well known, large-scale applications of detectors for muography involve aspects of data readout, processing and analysis that still offer room for improvements.

\section{References}

Acquafredda, R., et al. (2009). The OPERA experiment in the CERN to Gran Sasso neutrino beam, J. Instrum., 4, P04018.

Agafonova, N., et al. (2012). Momentum measurement by the Multiple Coulomb Scattering method in the OPERA lead emulsion target, New J. Phys., 14, 013026.

Agafonova, N., et al. (2014). Evidence for $v_{\mu} \rightarrow v_{\tau}$ appearance in the CNGS neutrino beam with the OPERA experiment, Phys. Rev. D., 89, 051102.

Aoki, S., K. Hoshino, M. Nakamura, K. Niu, K. Niwa and N. Torii (1990). Fully Automated Emulsion Analysis System, Nucl. Instrum. Meth. B, 51, 466-472.

Armenise, N., et al. (2005). High-speed particle tracking in nuclear emulsion by last-generation automated microscopes, Nucl. Instrum. Meth. A, 551, 261-270.

Arrabito, L., et al. (2006). Hardware performance of a scanning system for high speed analysis of nuclear emulsions, Nucl. Instrum. Meth. A, 568, 578-587.

Arrabito, L., et al. (2007). Track reconstruction in the emulsion-lead target of the OPERA experiment using the ESS microscope, 2, P05004.

Bozza, C., et al. (2013). An integrated system for large scale scanning of nuclear emulsions, Nucl. Instrum. Meth. A, 703, 204-212.

De Serio, M., et al. (2005). High precision measurements with nuclear emulsions using fast automated microscopes, Nucl. Instrum. Meth. A, 554, 247-254.

Eskut, E., et al. (1997). The CHORUS experiment to search for muon-neutrino $\rightarrow$ tau-neutrino oscilla- tion, Nucl. Instrum. Meth. A, 401, 7-44.

Eskut, E., et al. (2008). Final results from a search for $v_{\mu} \rightarrow v_{\tau}$ oscillations with the CHORUS experiment, Nucl. Phys. B, 793, 326-343.

Nakamura, T., et al. (2006). The OPERA film: New nuclear emulsion for large-scale, high-precision experiments, Nucl. Instrum. Meth. A, 556, 80-86.

Nishiyama, R., S. Miyamoto and N. Naganawa (2014). Experimental study of source of background noise in muon radiography using emulsion film detectors, Geosci. Instrum. Meth., 3, 29-39.

Niwa, K., K. Hoshino and K. Niu (1974). Auto scanning and measuring system for the emulsion chamber, In: Proceedings of the International Cosmic Ray Symposium on High Energy Phenomena, Cosmic Ray Laboratory, University of Tokyo, Japan, 149.

Rosa, G., A. Di Bartolomeo, G. Grella and G. Romano (1997). Automated analysis of digitized TV-images by a computer-driven optical microscope, Nucl. Instrum. Meth. A, 394, 357-367.

Tanaka, H.K.M., T. Nakano, S. Takahashi, J. Yoshida, M. Takeo, J. Oikawa, T. Ohminato, Y. Aoki, E. Koyama, H. Tsuji and K. Niwa (2007a). High resolution imaging in the inhomogeneous crust with cosmic-ray muon radiography: The density structure below the volcanic crater floor of Mt. Asama, Japan, Earth Planet. Sc. Lett., 263, 104-113.

Tanaka, H.K.M., T. Nakano, S. Takahashi, J. Yoshida, H. Ohshima, T. Maekawa, H. Watanabe and K. Niwa (2007b). Imaging the conduit size of the dome with cosmic-ray muons: The structure beneath Showa-Shinzan Lava Dome, Japan, Geophys. Res. Lett., 34, L22311.

${ }^{\star}$ Corresponding author: Cristiano Bozza, Università di Salerno, Dipartimento di Fisica "E. R. Caianiello", Salerno, Italy; email: cbozza@unisa.it.

C 2017 by the Istituto Nazionale di Geofisica e Vulcanologia. All rights reserved. 